\title{
Optical Performance Analysis of Conical Cavity Receiver
}

\author{
Hu Xiao' ${ }^{1}$, Yanping Zhang ${ }^{1}$, Chongzhe Zou ${ }^{1}$ \\ ${ }^{1}$ Huazhong University of Science and Technology \\ 1037 Luoyu road, Wuhan, China \\ 1061094586@qq.com; zyp2817@hust.edu.cn; zcz@hust.edu.cn
}

\begin{abstract}
In this paper, the commercial software TracePro was used to investigate the effects of some factors on a conical cavity receiver, such as the conical angle, the number of loops of the helical tube, and the distance between the focal point of the collector and the aperture. These factors affect the optical efficiency, the maximum heat flux density, and the light distribution in the conical cavity. The optical performance of the conical receiver was studied and analyzed using the Monte Carlo ray tracing method. The results showed that the amount of light rays reaching the helical tube increases with the increasing of the conical angle, while the optical efficiency decreases and the maximum heat flux density increases.
\end{abstract}

Keywords: Parabolic collector, Conical cavity receiver, Geometric parameters, Optical performance.

\section{Introduction}

Compared with other forms of solar thermal utilization, the solar parabolic dish system has the highest thermal efficiency, a concentration ratio of more than 2000, and an operating temperature of over $900 \mathrm{~K}[1,2]$. The boundary conditions of the inner surface of the cavity have often been simplified in different ways by many researchers. Flesch et al.[3] and Xiao et al.[4] did not consider the helical tube in the cavity when exploring the effects of wind on the thermal performance of the receiver. Their models were greatly simplified. Wu et al.[5,6] studied the convection and radiation loss of a fully open cylindrical cavity receiver. The inner surface of the cavity was regarded as a wall with a constant heat flux. In the numerical simulation of a hemispherical cavity receiver, Cui et al.[7] assumed that the wall temperature of the helical tube iwas constant. This assumption was based on the fact that the helical tube material has a good thermal conductivity. Compared to the work of Cui[7], it was more advisable to simplify similar models, as done by Reddy [8,9].

Based on some reasonable assumptions, the theoretical analysis methods were used to derive models for the heat flux distribution and optical loss of receivers [10,11].

Xie et al.Error! Reference source not found. performed optical simulations of cylindrical, conical and spherical cavity receivers. In the subsequent study of this paper, the conical receiver with a conical angle of $100^{\circ}$ was considered to have the highest thermal efficiency [13][14]. When investigating the optical performance of the cavity receiver, most researchers pay attention to the differences between the receivers of different geometries. However, there are not many further studies on the effects of critical geometric parameters of a receiver.

In this paper, the effects of the critical geometric parameters on the optical performance of conical cavity receivers are investigated .

\section{Physical model}

As shown in Fig. 1, the parabolic collector model and the cavity receiver model are established. A virtual circle is created as the light source plane, which emits light rays. Most of the dimensions are based on the experimental platform designed by our research group, as shown in Fig. 2. The parabolic collector is an ideal paraboloid with a diameter of $5060 \mathrm{~mm}$ and a focal length of $3200 \mathrm{~mm}$.

Figure 3(a) and (b) illustrates the cross section and the external view of the cavity receiver respectively. The thickness $(\sigma)$ of the thermal insulation layer is $75 \mathrm{~mm}$. The maximum diameter (dcav) of the cavity is $460 \mathrm{~mm}$. At the same time, the diameter (dap) of the aperture is $200 \mathrm{~mm}$ by reference to Refs. $[15,16]$ about the aperture size of a similar receiver model. The conical angle $(\beta)$ and the length $(\mathrm{L})$ of the cavity are varied. For the proposed model, different cavity lengths correspond 
to different numbers ( $\mathrm{n}$ ) of loops of the helical tube. In addition, the diameter (dt) of the helical tube is $42 \mathrm{~mm}$ and the wall thickness $(\delta \mathrm{t})$ is $3 \mathrm{~mm}$. In view of manufacturing errors, the pitch is set to $47 \mathrm{~mm}$, leaving a gap between each loop.

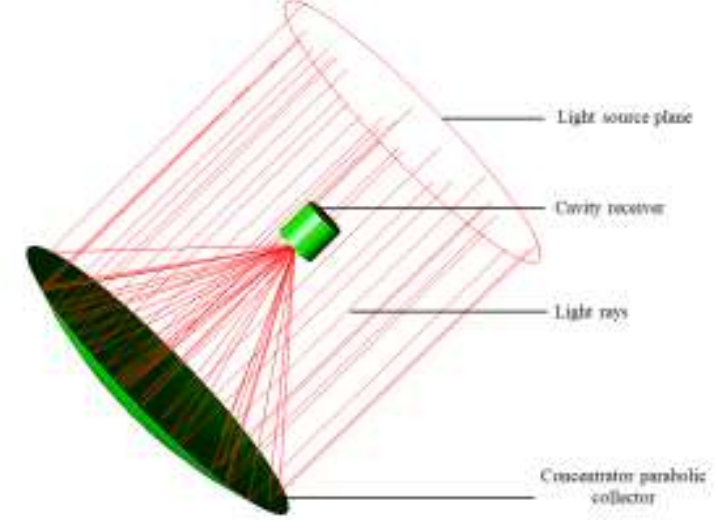

Fig. 1 Physicial model of optical section.

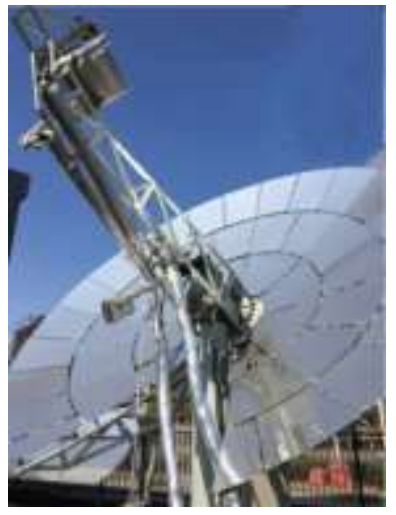

Fig. 2 Parabolic collector and cavity receiver.

To investigate the influence of the conical angle, receivers with 6 loops are studied as the conical angle varies from $0^{\circ}$ to $15^{\circ}$. When the above two parameters are discussed, the position of the receiver is changed. That is, the distance (d) between the focal point of the collector and the aperture varies from $-120 \mathrm{~mm}$ to $150 \mathrm{~mm}$. A distance of less than $0 \mathrm{~mm}$ means that the focal point is inside the cavity. A distance of more than $0 \mathrm{~mm}$ means that the focal point is outside the cavity, as shown in Fig. 3(a).

When the conical angle is $15^{\circ}$ and the number of loops is 8 in the proposed model, the minimum winding radius of the helical tube is approximately $90 \mathrm{~mm}$. As a comparison, the diameter of the tube is $42 \mathrm{~mm}$. Referring to the experiment made by Prakash et al. [17], a small winding radius leads to a large manufacturing stress and residual stress. Then, it increases the difficulty of winding. To avoid an overly small winding radius, a larger conical angle and a larger number of loops are avoided. When the number of loops is less than 5, the working fluid in the tube may not be sufficiently heated. Therefore, this option is not considered. When the distance between the focus point and the aperture varies from $-120 \mathrm{~mm}$ to $150 \mathrm{~mm}$, it is ensured that most of the light rays enter the cavity.

\section{Optical efficiency analysis}

Figures 5 and 6 reflect the variation of optical efficiency for different conical angles and different numbers of loops when the distance between the focus point and the aperture varies, respectively. It is observed that the optical efficiency first rises sharply as the distance increases. Then, it remains relatively stable, and finally drops sharply. The corresponding three situations are demonstrated in Fig.7. Figure 7(a) illustrates the case where the distance is less than $0 \mathrm{~mm}$, and the focus point is inside the cavity. At this time, the optical efficiency is low because a large number of rays are blocked by the front cover of the receiver. In Fig. 7(b), the focal point is near the aperture, and most rays can enter the cavity. Thus, the optical efficiency is high. Figure 7(c) shows the case where the distance is greater than $0 \mathrm{~mm}$, and the focal point is outside the cavity. At this time, the optical efficiency is low because a portion of the rays is blocked again. The maximum optical efficiency occurs when the distance is $-60 \mathrm{~mm}$.

A comparison of the four curves in Fig. 5 indicates that the optical efficiency decreases with increasing conical angle. This phenomenon can be explained by the size of the absorption area in the cavity. When the conical angle is small, the surface area of the cavity is large. After light rays enter the cavity, they reflect more between the surfaces in the cavity. Therefore, the energy can be fully absorbed and the optical efficiency is high. The maximum optical efficiency occurs when the conical angle is $0^{\circ}$.

The four curves in Fig. 6 exhibits that the optical efficiency increases slightly as the number of loops increases. This result can also be attributed to the size of the absorption area in the cavity. Figure 8 presents a cross-sectional view of 
the receiver for different numbers of loops. The larger number of loops means greater lengths and greater surface area. Light energy can be more fully absorbed in the cavity, resulting in an increase in optical efficiency. In addition, when the number of loops is sufficiently large, its continued increase does not effectively increase the optical efficiency. Thus, the curves for 7 and 8 loops are almost coincident in Fig. 6(a) and 6(b). The maximum optical efficiency occurs when the number of loops is 8 .

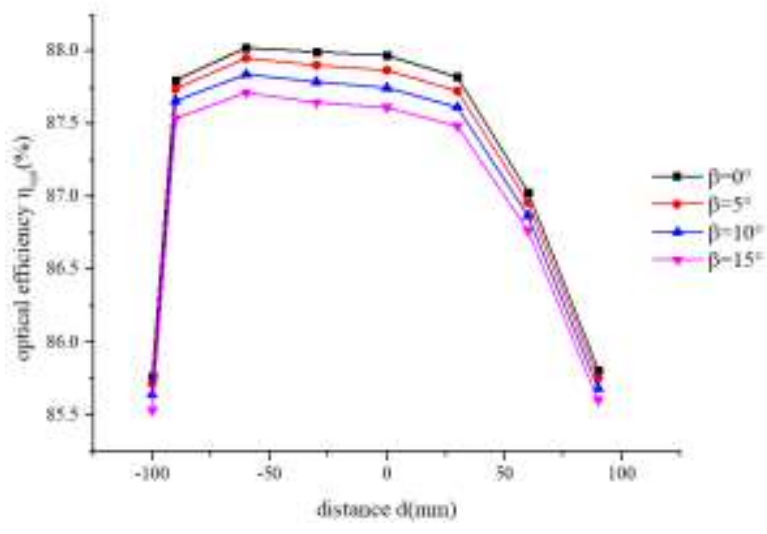

Fig. 5 Relationship between the optical efficiency and the distance between the focusal point and the aperture for different conical angles.

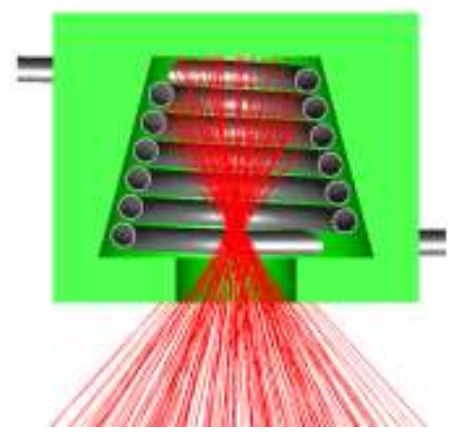

(a) $\mathrm{d}=-120 \mathrm{~mm}$

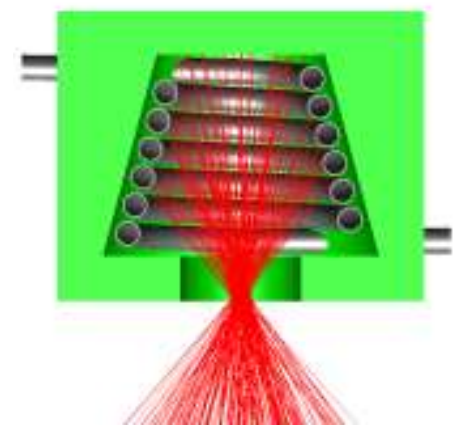

(b) $\mathrm{d}=0 \mathrm{~mm}$;

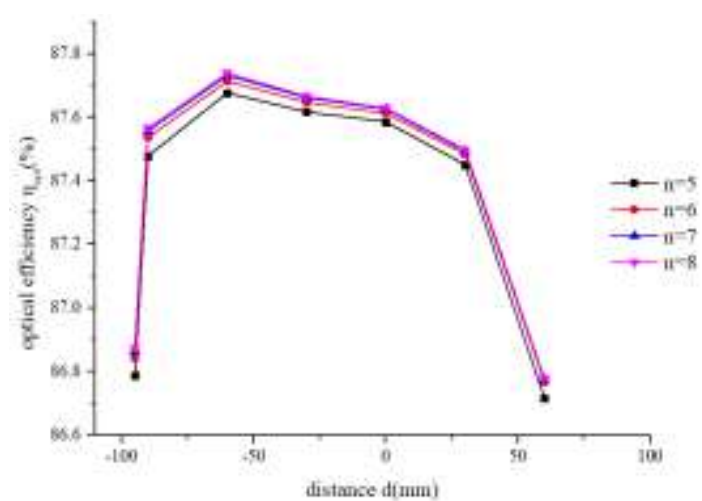

Fig. 6 Relationship between the optical efficiency and the distance between the focusal point and the aperture for different numbers of loops.

Fig. 7 Light distributions underat different distances between the focusal point and the aperture.

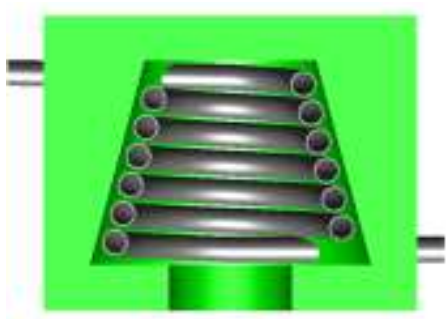

(a) $n=6$

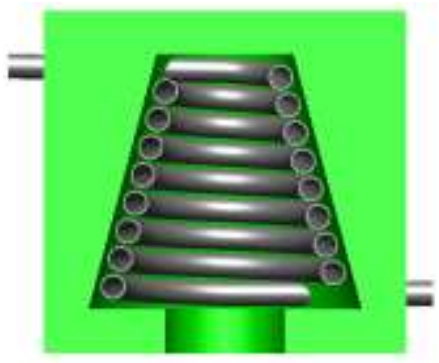

(b) $n=8$

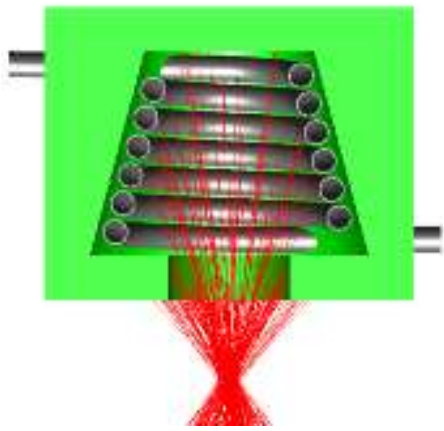

(c) $\mathrm{d}=120 \mathrm{~mm}$

Fig. 8 Cross-section of the receiver or different numbers of loops. 
A comparison of the curves in Figs. 5 and 6 suggests that the maximum difference in optical efficiency for the different conical angles is approximately $0.5 \%$ and approximately $0.1 \%$ for different numbers of loops. It is concluded that the effect of conical angle on optical efficiency is significantly greater than the effect of the number of loops.

\section{Conclusion}

In this paper, three factors affecting the optical performance are investigated. They are the number of loops, the conical angle, and the distance between the focal point and the aperture when the diameter of the aperture, the incident light conditions and the material are constant. The study of this paper showed that for a conical cavity receiver, the optical efficiency increases with the decrease of the conical angle and the increase of the number of loops.

\section{References}

[1] Tsoutsos T, Gekas V, Marketaki K. "Technical and economical evaluation of solar thermal power generation," Renew energ, vol. 28, no. 6, pp.873-886, 2003.

[2] Huang W, Huang F, Hu P, et al. "Prediction and optimization of the performance of parabolic solar dish concentrator with sphere receiver using analytical function," Renew energ, vol. 53, no. 9, pp. 18-26, 2013.

[3] Flesch R, Stadler H, Uhlig R, et al. "Numerical analysis of the influence of inclination angle and wind on the heat losses of cavity receivers for solar thermal power towers," Sol Energy, vol. 110, pp. 427-437, 2014.

[4] Xiao L, Wu S Y, Li Y R. "Numerical study on combined free-forced convection heat loss of solar cavity receiver under wind environments," Int. J. Therm. Sci., vol.60, no.1, pp. 182-194, 2012.

[5] Wu S Y, Guo F H, Xiao L. "Numerical investigation on combined natural convection and radiation heat losses in one side open cylindrical cavity with constant heat flux," Int J Heat Mass Tran., vol. 71, no. 3, pp. 573-584, 2014

[6] Wu S Y, Xiao L, Li Y R. "Effect of aperture position and size on natural convection heat loss of a solar heat-pipe receiver," Appl. Therm. Eng., vol. 31,no. 14-15, pp. 2787-2796, 2011.

[7] Cui F, He Y, Cheng Z, et al. "Study on combined heat loss of a dish receiver with quartz glass cover," Appl. Energ., vol. 112, no. 4,pp.690-696, 2013.

[8] Reddy K S, Vikram T S, "Veershetty G. Combined heat loss analysis of solar parabolic dish- modified cavity receiver for superheated steam generation," Sol. Energy, vol. 121, pp. 78-93, 2015.

[9] Vikram T S, Reddy K S. "Estimation of heat losses from modified cavity mono-tube boiler receiver of solar parabolic dish for steam generation," Energy Procedia, vol. 57, pp. 371-380, 2014.

[10] Collado F J. "One-point fitting of the flux density produced by a heliostat," Sol. Energy, vol. 84, no.4, pp. 673-684, 2010

[11] Li H, Huang W, Huang F, et al. "Optical analysis and optimization of parabolic dish solar concentrator with a cavity receiver," Sol. Energy, vol. 92, no.4, pp.288-297, 2013.

[12] Xie W T, Dai Y J, Wang R Z. "Numerical and experimental analysis of a point focus solar collector using high concentration imaging PMMA Fresnel lens," Energ. Convers. Manage., vol. 52, no. 6, pp. 2417-2426, 2011.

[13] Li X, Dai Y J, Wang R Z. "Performance investigation on solar thermal conversion of a conical cavity receiver employing a beam-down solar tower concentrator," Sol. Energy, vol. 114, pp. 134-151, 2015.

[14] DaaboA M, Mahmoud S, Al-Dadah R K. "The optical efficiency of three different geometries of a small scale cavity receiver for concentrated solar applications," Appl. Energ., vol. 179, pp. 1081-1096, 2016.

[15] Zou C, Zhang Y, Feng H, et al. "Effects of geometric parameters on thermal performance for a cylindrical solar receiver using a 3D numerical model," Energ. Convers. Manage., vol. 149, pp. 293-302, 2017.

[16] Zou C, Zhang Y, Falcoz Q, et al. "Design and optimization of a high-temperature cavity receiver for a solar energy cascade utilization system," Renew energ, vol. 103, pp. 478-489, 2017

[17] Prakash M, Kedare S B, Nayak J K. "Investigations on heat losses from a solar cavity receiver," Sol. Energy, vol. 83, no. 2, pp. 157-170, 2009. 